\title{
A Case of Hypercalcemia and Gastric Necrosis from Hot Pack Ingestion
}

\author{
Pradeep Padmanabhan • Henry A. Spiller • \\ George M. Bosse
}

Published online: 8 April 2010

(C) American College of Medical Toxicology 2010

\begin{abstract}
Hot packs (instant hot compresses) are frequently used to relieve pain. We report a patient who had significant complications from ingestion of a hot pack containing calcium salts. A 35-year-old male swallowed three hot packs, and developed hematemesis, severe abdominal pain, and hypercalcemia $(21.1 \mathrm{mg} / \mathrm{dl})$. He developed diffuse gastric necrosis requiring gastrectomy and colonic interposition. Hypercalcemia was treated with intravenous fluids, pamidronate, and calcitonin. A Medline search revealed no prior report on hot pack ingestion though ingestion of calcium salts has been reported. Hot packs can potentially cause significant injury both from an exothermic reaction and hypercalcemia. Ingestions of calcium salts can result in necrosis of the stomach. Management includes aggressive treatment of hypercalcemia, supportive care and upper gastrointestinal endoscopy.
\end{abstract}

Keywords Hypercalcemia · Hot pack · Thermal injury · Gastrointestinal necrosis · Gastric ulcers

\footnotetext{
P. Padmanabhan $(\bowtie)$

Department of Pediatrics, Division of Pediatric Emergency

Medicine, Kosair Children's Hospital, University of Louisville,

571 S. Floyd Street, Ste 300,

Louisville, KY 40202, USA

e-mail: p0padm01@gwise.louisville.edu

H. A. Spiller

Kentucky Poison Center,

Louisville, KY, USA

G. M. Bosse

Division of Emergency Medicine, University of Louisville, Louisville, KY, USA
}

\section{Introduction}

Hot packs (instant hot compresses) are utilized frequently to alleviate pain caused by various ailments. The ingredients of hot packs vary and may include calcium salts, sodium, potassium, strontium, iron, magnesium, activated charcoal, vermiculite, sodium thiosulfate, menthol, and capsicum extract. Hot packs are widely available in supermarkets and drugstores. A review of the medical literature from 1956 to 2008 revealed no case reports of hot pack ingestion. We present an unusual case of a patient who ingested hot packs and suffered serious injury requiring total gastrectomy with multiple complications and hospitalizations.

\section{Case Report}

A 35-year-old male presented to the emergency department (ED) after admitting to ingesting three hot packs while he was in jail. His past history was significant for a seizure disorder, alcoholism, and marijuana use and his medications included phenytoin and mirtazapine. The hot packs were given to him to alleviate back pain. At the jail, he was in medical isolation due to possibly infective "spider bite" skin lesions, and hence, did not have access to any other chemicals or medications. He stated that he consumed the packets so that he could come out of medical isolation. It was not known if he had broken open all of the packets before consumption, although he admitted to drinking some of the contents as a solution. He denied any suicidal ideations. He developed severe abdominal pain and approximately 20 episodes of "red colored" emesis (color of the ingredients in hot packs). He reported his symptoms to the prison authorities approximately $5 \mathrm{~h}$ after ingestion. However, it is unclear when his symptoms began. He also 
had mid-anterior chest pain worsening after deep inspiration. He arrived in the emergency room approximately $6 \mathrm{~h}$ post-ingestion.

Physical examination revealed an alert male in significant distress secondary to severe abdominal pain with a temperature of $98^{\circ} \mathrm{F}$, heart rate of $90 \mathrm{bpm}$, blood pressure of $130 / 75 \mathrm{~mm} \mathrm{Hg}$, respiratory rate of 18 breaths per min, and a room air oxygen saturation of $98 \%$. The Gastroccult ${ }^{\circledR}$ test on the vomitus was positive. He had no obvious skin lesions except redness to the tip of his nose suggestive of cellulitis. The oropharyngeal exam revealed no mucosal burns and the chest was clear to auscultation. He had significant tenderness along the left side of the chest wall and diffusely over the upper abdomen with guarding, but no rebound tenderness was present. Bowel sounds were present. Stool Hemoccult ${ }^{\circledR}$ test was negative. The cardiovascular and central nervous systems were normal on exam.

Total white blood cell count was noted to be $27,500 / \mathrm{mm}^{3}$ with hemoglobin of $16 \mathrm{~g} / \mathrm{dl}$. The metabolic panel included total serum calcium $21.1 \mathrm{mg} / \mathrm{dl}$, phosphorus $2.3 \mathrm{mg} / \mathrm{dl}$, magnesium $1.4 \mathrm{mg} / \mathrm{dl}$, sodium $147 \mathrm{~m} \mathrm{Eq} / \mathrm{L}$, potassium $4.1 \mathrm{~m} \mathrm{Eq} / \mathrm{L}$, chloride $112 \mathrm{~m} \mathrm{Eq} / \mathrm{L}$, bicarbonate $19 \mathrm{~m} \mathrm{Eq} / \mathrm{L}$, glucose $149 \mathrm{mg} / \mathrm{dl}$, blood urea nitrogen $9 \mathrm{mg} / \mathrm{dl}$, and a serum creatinine of $0.9 \mathrm{mg} / \mathrm{dl}$. His electrocardiogram demonstrated a normal sinus rhythm with a corrected QT interval of $0.373 \mathrm{~s}$ which did not change significantly on subsequent electrocardiograms during his hospital stay. Chest X-ray was negative. An abdominal X-ray performed the next day revealed three radio opaque deposits in the right lower quadrant, suggestive of calcium deposits.

He was hospitalized, and received intravenous fluids, along with intravenous pamidronate (90 mg over $24 \mathrm{~h}$ ), intravenous calcitonin 4 units $/ \mathrm{kg} \times 4$ doses every $12 \mathrm{~h}$, oral diltiazem $30 \mathrm{mg}$ every $6 \mathrm{~h}$, and a single dose of intravenous magnesium sulfate $2 \mathrm{~g}$. His mental status remained normal, and no dysrhythmias were noted during the hospital stay. However, he continued to have significant abdominal pain requiring multiple doses of morphine sulfate. His calcium level dropped to $10.1 \mathrm{mg} / \mathrm{dl}$ after $21 \mathrm{~h}$, and his phosphorus rose to $4.1 \mathrm{mg} / \mathrm{dl}$.

Upper gastrointestinal endoscopy was performed the day after presentation. The endoscopy findings included diffuse esophagitis, deep antral ulceration, and an ischemic appearing stomach visualized in Figs. 1, 2, 3, and 4. His condition worsened during the next $48 \mathrm{~h}$ with increasing pain and continued vomiting requiring narcotics and fluid resuscitation. However, the hematemesis resolved, and even though the hemoglobin values decreased from $16 \mathrm{gm} / \mathrm{dl}$ to $11.7 \mathrm{gm} / \mathrm{dl}$, the decline was not significant to warrant transfusions. He remained hemodynamically stable subsequently during his stay in the hospital. He was placed on total parenteral nutrition. As he continued to have severe pain and frequent vomiting, he underwent repeat endoscopy

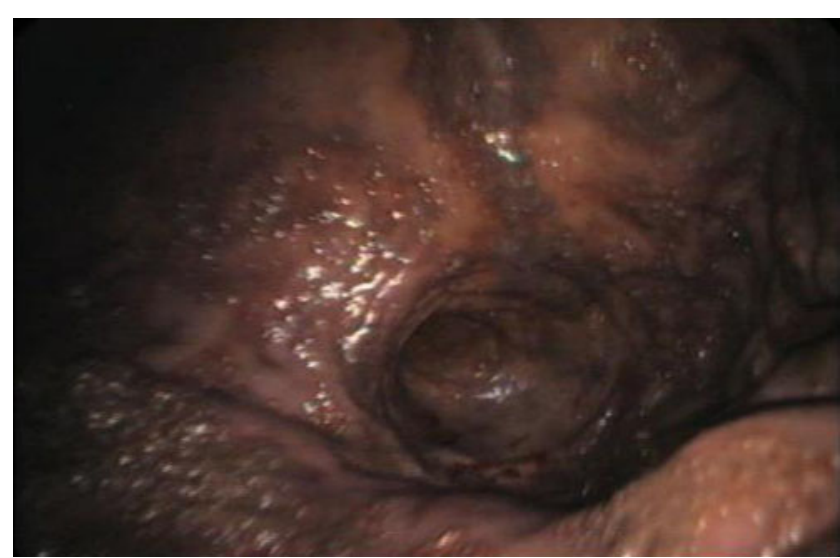

Fig. 1 Diffuse edema and ischemia of gastric mucosa

which demonstrated diffuse necrosis and friability of the stomach. A total gastrectomy and gastrointestinal tract reconstruction was performed 2 weeks after the initial ED presentation. He has been hospitalized on multiple occasions since due to complications including intra-abdominal abscesses, septic shock, and has undergone several surgical procedures. His most recent hospitalization revealed a bronchoenteric fistula (fistula between bronchus to the esophagocolonic anastamosis) which has restricted him to total parenteral nutrition.

\section{Discussion}

The contents of the instant hot pack that our patient ingested include calcium chloride $90-97 \%$, sodium chloride $1-2 \%$, potassium chloride $2-3 \%$, strontium chloride $0-1 \%$, and water. Potential complications from hot pack ingestion include direct thermal injury, local tissue irritation, hypercalcemia, hypomagnesemia, and hyperchloremic acidosis.

The thermal injury from the hot pack depends on the time of activation of the contents of the pack. An exothermic reaction is a chemical reaction where heat is liberated in contrast to an endothermic reaction where heat is absorbed [1]. The salts (e.g., calcium chloride) when in contact with water form new ionic interactions and release thermal energy as a result of these interactions. The instant hot pack that this patient ingested liberates heat through an exothermic reaction when calcium chloride in the pack comes into contact with water, and occurs when the separate inner compartments are broken down by squeezing the pack. Activation of hot packs can generate temperatures up to $130^{\circ} \mathrm{F}$ under certain conditions [2]. Temperature (both heat and cold) can significantly change pyloro-antral motility, thus causing retardation of transpyloric flow, thereby increasing the likelihood of prolonged injury [3]. Animal experiments have shown that local heat causes disruption in vascular flow, initially causing destruction of 


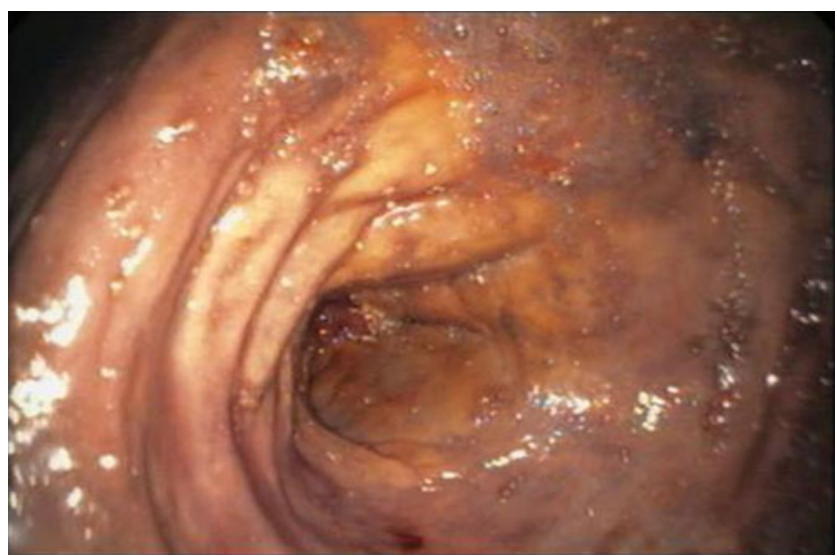

Fig. 2 Whitish plaques with diffuse ischemia at the gastric antrum

the smaller capillaries followed by destruction of larger blood vessels in the stomach [4].

Concentrated calcium salts can cause severe local tissue damage and significant systemic consequences. The severe gastric injury in our patient was more likely due to direct contact from concentrated calcium salts from the hot pack. Instances of skin and soft tissue necrosis from contact with calcium chloride have been reported [5]. Accidental extravasations during intravenous administration of calcium chloride have been linked to skin and tissue necrosis (calcinosis cutis) [6]. "Drill Mud," which contains calcium chloride, has resulted in necrotic skin lesions among coal miners and oil drillers [7]. Skin lesions such as deep dermal necrosis from exposure to calcium salts may sometimes be mistaken for child abuse or burns [8]. Hypercalcemia has also been associated with upper gastrointestinal bleeding [9]. If our patient had ingested the contents without mixing with water, the exothermic reaction may have occurred in the stomach producing immense heat with the potential for immediate devastating injury. Since he ingested some of the contents of the hot pack as a solution and had no obvious oropharyngeal lesions, the necrosis of the stomach was

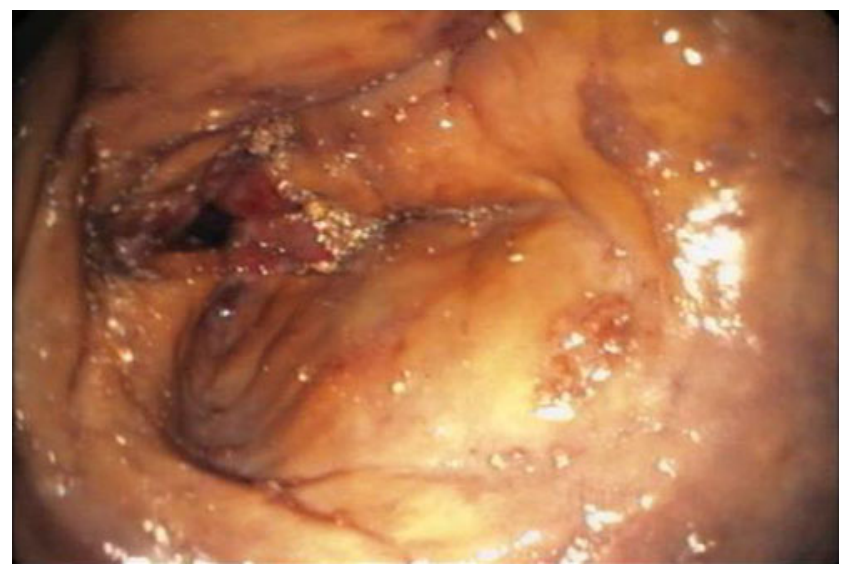

Fig. 3 Ulcerations and erosions surrounding the pyloric opening

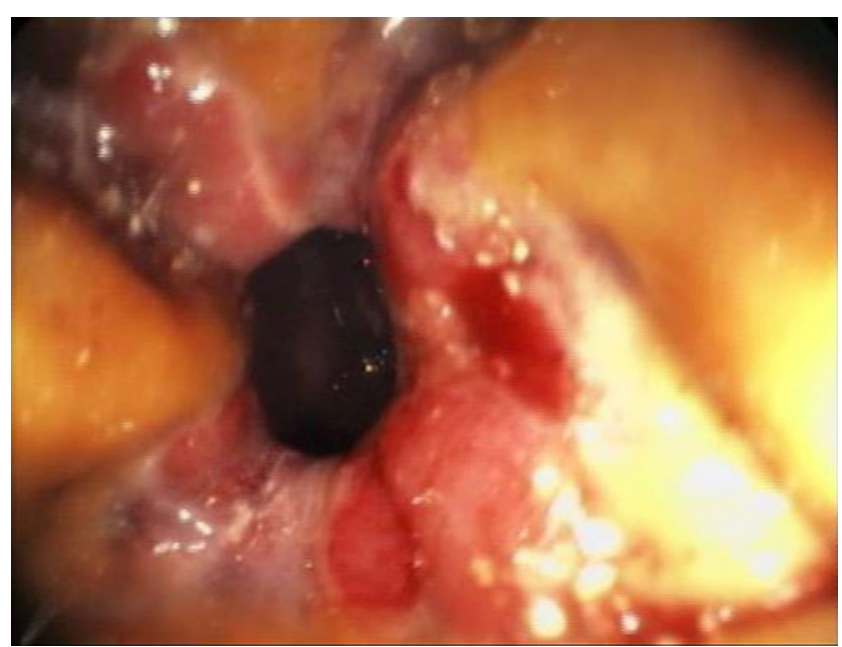

Fig. 4 Deep ulcerations at the pyloric opening

perhaps less likely from thermal injury and was more likely from the direct tissue injury from calcium salts.

For hypercalcemia to develop, the normal calcium regulation system must be overwhelmed by an excess of parathyroid hormone, calcitriol, or a massive exogenous calcium load. Systemic hypercalcemia can result in fatigue, depression, confusion, vomiting, constipation, reversible renal tubular defects, increased urination, bradycardia, atrioventricular block, and a short QT interval on electrocardiogram [10]. Even though our patient developed significant hypercalcemia, he did not manifest clinical side effects of hypercalcemia. Even at levels greater than $12 \mathrm{mg} / \mathrm{dl}$, patients have been reported to be asymptomatic except for vomiting [11]. The lack of systemic manifestations could perhaps be attributed to the acute rise in calcium levels with insufficient time for distribution into the tissues. There is no clear correlation between levels of calcium and the severity of symptoms. However, severe hypercalcemia may result in coma and serious dysrhythmias resulting in cardiac arrest [12].

In our patient, the elevated serum calcium suggests that there was significant absorption of unbound ionized calcium due to the large amount and form of calcium salts ingested. In addition, the chloride which is released from these salts may then be absorbed causing hyperchloremic acidosis by decreasing the reabsorption of bicarbonate [13]. The patient's serum chloride levels were slightly above normal and may have been secondary to absorption of chloride from the hot pack.

Several therapies can be utilized to treat hypercalcemia. Our patient received intravenous fluid resuscitation, pamidronate, and diuresis (furosemide). Hydration lowers calcium via dilution, corrects prerenal azotemia, and corrects dehydration commonly associated with hypercalcemia [10]. Diuresis with loop diuretics given after volume resuscitation improves hypercalcemia by enhancing calciu- 
ria [12]. High urinary sodium excretion also promotes urinary calcium excretion. Pamidronate is a bone resorption inhibitor, and is used primarily in hypercalcemia associated with malignancy. Pamidronate is perhaps not indicated in this patient even though its efficacy in correcting a case of intractable hypercalcemia from oral vitamin D intoxication has been reported [14]. It reduces bone turnover and when used in combination with adequate hydration to increase renal excretion of calcium, reduces serum calcium concentrations. In the kidney, calcitonin increases the excretion of filtered phosphate, calcium, and sodium by decreasing their tubular reabsorption.

Under dietary conditions, strontium $(<1 \%$ in the hot pack) is poorly absorbed, with approximately $20 \%$ bioavailability and ultimately gets deposited in the bone [15]. Acute toxicity was not observed in a single case of large ingestion of strontium ferrite [16]. The primary impact of excess stable strontium appears to be a reduction of bone formation. Since it was present in minute quantities in the hot pack we have described, we do not believe that it had a role in the injury sustained by this patient.

In summary, we report an unusual case of hypercalcemia, hypomagnesemia, esophagitis, gastritis, and gastric necrosis requiring complete gastrectomy associated with ingestion of a calcium salt containing hot pack.

\section{References}

1. Daintith J (2008) Oxford dictionary of chemistry, 6th edn. Oxford University Press, New York, p 216
2. Raleigh G, Rivard R, Fabus S (2005) Air-activated chemical warming devices: effects of oxygen and pressure. Undersea and Hyperb Med 32(6):445

3. Sun WM, Penagini R, Hebbard G (1995) Effect of drink temperature on antropyloroduodenal motility and gastric electrical activity in humans. Gut 37(3):329-334

4. Falk P (1983) The effect of elevated temperature on the vasculature of mouse jejunum. Br J Radiol 56(661):41-49

5. Min P, Kim VJ, Raho JM (2007) Skin and soft tissue necrosis from calcium chloride in a deicer. J Emerg Med 32(1):41-44

6. Kagen MH, Bansal MG, Grossman M (2000) Calcinosis cutis following the administration of intravenous calcium therapy. Cutis 65:193-194

7. Wheeland RG, Roundtree JM (1985) Calcinosis cutis from percutaneous penetration and deposition of calcium. J Am Acad Dermatol 12:172-175

8. Zurbuchen P, Lecoulte C, Calza AM, Halperin DS (1996) Cutaneous necrosis after contact with calcium chloride: a mistaken diagnosis of child abuse. Pediatrics 97:257-283

9. Saylor J, Sarosdy RP, Wright MF (1984) Hypercalcemia induced upper gastrointestinal bleeding after renal transplantation. Urology 24(4):337-339

10. Harrison's Principles of Internal Medicine (17th Ed). 2008. Chapter 347. p 2382

11. Slattery A, King WD, Nichols M, Fowler J (1995) Hypercalcemia following Damp-rid ${ }^{\mathrm{TM}}$ ingestion. Clin Toxicol 33:487

12. Williams Textbook of Endocrinology (11th ed). 2008. Chapter 27. p 1240

13. Rudolph's Pediatrics (21st Ed). 2003. Chapter 21.4.4. p 655

14. Chatterjee M, Speiser PW (2007) Pamidronate treatment of hypercalcemia caused by vitamin D toxicity. Journal Pediatr Endocrinol 20(11):1241-1248

15. Dorsey AF, Fransen ME, Diamond GL, et al. Toxicologic profile for strontium. Agency for Toxic Substances and Disease Registry (ATSDR), Atlanta, GA, USA (2004) US Department of Health and Human Services, Public Health Service

16. Kirrane BM, Nelson LS, Hoffman RS (2006) Massive strontium ferrite ingestion without acute toxicity. Basic Clin Pharmacol Toxicol 99(5):358-359 